\title{
Collecting Infectious Disease Data from LARS and Improving Data Quality in Taiwan
}

\author{
Chih-Ting Yeh*, Chia-Lin Li, Chih-Jung Ke and Chi-Ming Chang
}

Epidemic Intelligence Center, Centers for Disease Control, Taipei City, Taiwan

\section{Objective}

To improve data quality and sustain a good quality data collected by Laboratory Automated Reporting System (LARS), we use a Threestage Data Quality Correction (3DQC) strategy to ensure data accuracy.

\section{Introduction}

To immediately monitor disease outbreaks, the application of laboratory-based surveillance is more popular in recent years. Taiwan Centers for Disease Control (TCDC) has developed LARS to collect the laboratory-confirmed cases caused by any of 20 pathogens daily via automated submitting of reports from hospital laboratory information system (LIS) to LARS since 2014 [1]. LOINC is used as standardized format for messaging inspection data [1,2]. There are 37 hospitals have joined LARS, coverage rate about $59 \%$ of all hospitals in Taiwan. Recently, more than 10,000 of data are collected weekly and used in monitoring pathogen activity [3]. Therefore, it is important to ensure data quality that the data will lead to valuable information for public health surveillance.

\section{Methods}

A 3DQC strategy was designed to improve data accuracy and carried out by teamwork among TCDC, Taiwan Association for Medical Informatics (TAMI) and IT Company (Figure 1). In the first stage of 3DQC, IT Company checked data format. In the second stage, TCDC verified information between hospital inspection reports and data receiving in LARS. In the third stage, TAMI evaluated LOINC mapping and TCDC monitored stability of data transmission. After correcting the data, hospitals were approved to join LARS.

\section{Results}

During the first stage of 3DQC, we observed that some problems with syntax error in data (e.g. incorrect patient identification number, or lack of residence codes). Because some data were stored in Hospital information system (HIS) but not in LIS, an error may occur while hospital accessed records from HIS. In the second stage, $50-70 \%$ of inspection reports provided by each hospital had problems with semantic information error. For example, a positive result of influenza A on a screening flu test recorded in LIS but hospital transferred the wrong result with influenza B positive into LARS. In the third stage, we found that $20-30 \%$ of terms mismatched to LOINC code. This study categorized these terms into two groups (1) the Exception codes, which were considered reasonable and (2) the Error codes, and also reviewed Error codes and made a modified advice for hospitals to improve LOINC mapping. Through 3DQC strategy, the LOINC mapping rate raised from 40 to $80 \%$, Exception codes mapping was $20 \%$, and the total mapping rate was near $95-99 \%$ (Figure 2 ). So far, most hospitals have maintained a good quality data even they formally participate in LARS.

\section{Conclusions}

This study suggested that $3 \mathrm{DQC}$ can effectively detect problems and reduce errors of data collected from LARS, and indicated that effect of 3DQC can be maintained even hospital formally participates in LARS. Future research will focus on development of automatic programming of 3DQC to ensure high-quality data.

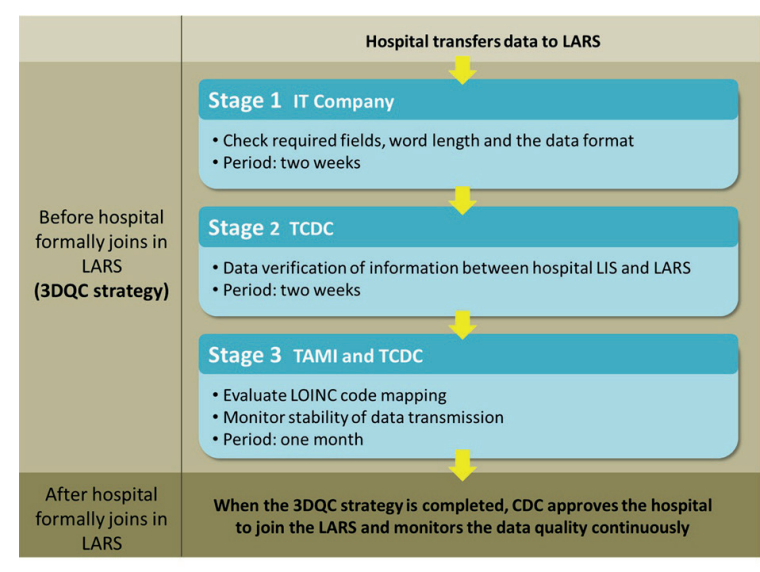

Figure 1. A Three-stage Data Quality Correction strategy

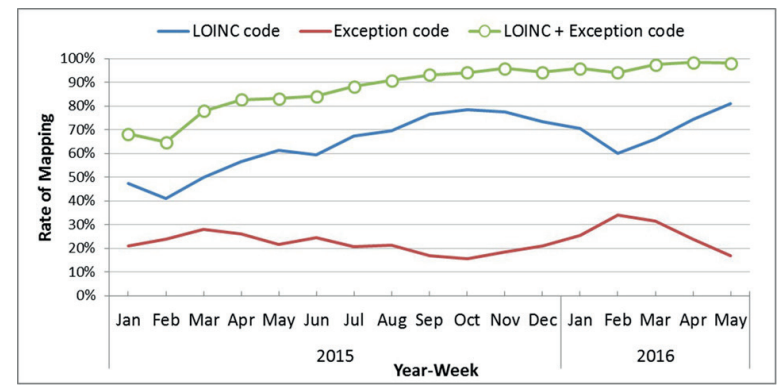

Figure 2. Rate of LOINC Mapping, 2015-2016

\section{Keywords}

laboratory automated reporting system; three-stage data quality correction; surveillance

\section{Acknowledgments}

We thank all the collaborating hospitals for providing data and cooperating with data correction, and also thank Mr. Chih-Yang Yeh from TAMI for providing expert advice for LOINC mapping.

\section{References}

1. Chang CM, Kuo HW, Ye YW, Liu YL, Chuang JH. Implementation of a Nationwide Automated Laboratory Reporting System for Infectious Disease Surveillance Based On the Epidemic Information Exchange Platform and the Use of LOINC Standard. JTAMI. 2015 Mar; 24(1):1-10.

2. McDonald CJ, Huff SM, Suico JG, Hill G, Leavelle D, Aller R, Forrey A, Mercer K, DeMoor G, Hook J, Williams W, Case J, Maloney P. LOINC, a universal standard for identifying laboratory observations: a 5-year update. Clin Chem. 2003 Apr;49(4):624-33.

3. Kuo HW, Chang CM, Ke CJ, Liu YL, Liu DP. Apply Laboratory Automated Reporting System on Infectious Disease Surveullance. JTAMI. 2016 Mar; 25(1):13-22.

\section{${ }^{*}$ Chih-Ting Yeh}

E-mail: emilyyeh@cdc.gov.tw 